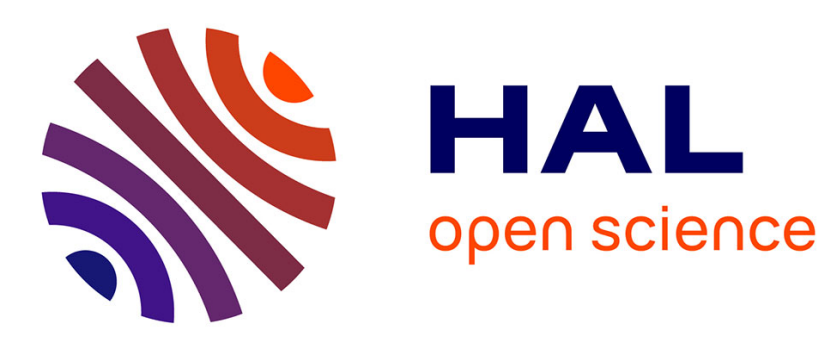

\title{
An efficient data model for energy prediction using wireless sensors
}

\author{
Michel Chammas, Abdallah Makhoul, Jacques Demerjian
}

\section{To cite this version:}

Michel Chammas, Abdallah Makhoul, Jacques Demerjian. An efficient data model for energy prediction using wireless sensors. Computers and Electrical Engineering, 2019, 76, pp.249 - 257. hal02182805

\section{HAL Id: hal-02182805 \\ https://hal.science/hal-02182805}

Submitted on 13 Jul 2019

HAL is a multi-disciplinary open access archive for the deposit and dissemination of scientific research documents, whether they are published or not. The documents may come from teaching and research institutions in France or abroad, or from public or private research centers.
L'archive ouverte pluridisciplinaire HAL, est destinée au dépôt et à la diffusion de documents scientifiques de niveau recherche, publiés ou non, émanant des établissements d'enseignement et de recherche français ou étrangers, des laboratoires publics ou privés. 


\title{
An Efficient Data Model for Energy Prediction using Wireless Sensors
}

\author{
Michel Chammas $^{\mathrm{a}}$, Abdallah Makhoul ${ }^{\mathrm{b}}$, Jacques Demerjian $^{\mathrm{c}}$ \\ ${ }^{a}$ Digital Humanities Center, University of Balamand, El-Koura, Lebanon \\ ${ }^{b}$ Femto-ST Institute, UMR CNRS 6174, Université de Bourgogne Franche-Comté, Besançon, \\ France \\ ${ }^{c}$ LARIFA-EDST, Faculty of Sciences, Lebanese University, Fanar, Lebanon
}

\begin{abstract}
Energy prediction is in high importance for smart homes and smart cities, since it helps reduce power consumption and provides better energy and cost savings. Many algorithms have been used for predicting energy consumption using data collected from Internet of Things (IoT) devices and wireless sensors. In this paper, we propose a system based on Multilayer Perceptron (MLP) to predict energy consumption of a building using collected information (e.g., light energy, day of the week, humidity, temperature, etc.) from a Wireless Sensor Network (WSN). We compare our system against four other classification algorithms, namely: Linear Regression (LR), Support Vector Machine (SVM), Gradient Boosting Machine (GBM) and Random Forest (RF). We achieve state-of-the-art results with $64 \%$ of the coefficient of Determination $R^{2}, 59.84 \%$ Root Mean Square Error (RMSE), 27.28\% Mean Absolute Error (MAE) and 27.09\% Mean Absolute Percentage Error (MAPE) in the testing set when using weather and temporal data.
\end{abstract}

Keywords: Energy Prediction, Multilayer Percetron (MLP), Data Mining,

Email addresses: michel.chammas@balamand.edu.lb (Michel Chammas), abdallah.makhoul@univ-fcomte.fr (Abdallah Makhoul), jacques.demerjian@ul.edu.lb (Jacques Demerjian)

Preprint submitted to Journal of ${ }^{A} T_{E} X$ Templates

April 15, 2019 
Machine learning, classification algorithms

\section{Introduction}

Smart Home technologies are among the most emerging trends in Internet of Things (IoT) today. Latest studies show the intelligence of smart devices and sensor networks in improving the quality of people's life [1]. IoT devices are

5 being used to collect data in order to analyze the behaviour and proper uses of energy. Whereas energy consumption has always been an important issue as the number of devices using electrical power are increasing. Since the mid 80 's, researchers mentioned the importance of energy forecasting in the future of smart buildings as it plays an essential role in energy and cost saving. Whereas buildings represent around $40 \%$ of the world energy usage, they are considered as the most cost-effective areas to reduce energy consumption. [2]. Therefore, this issue created an important challenge for the researchers to analyze the energy consumption in order to reduce it. Many studies have concluded that weather is one of several conditions that could affect the energy usage in a building [3], and weather data performs an important role for energy prediction and performance assessment of smart buildings and urban environments [4.

Different models have been used to predict power consumption like Autoregressive Integrated Moving Average model (ARIMA) [5], simple and multiple Linear Regression, Neuro-Fuzzy model [6], Support Vector Regression, Support 20 Vector Machines (SVM) [7, Artficial Neural Network (ANN) 8, Time-Series [9], or a combination of regression, Nearest Neighbor and ANN, whereas ANN has been considered to achieve the best results for energy prediction. Based on the statistics mentioned in [4, an overall of $47 \%$ of the energy consumption prediction models utilized ANN as machine learning algorithms, while $25 \%$ used SVM, $4 \%$ decision trees and $24 \%$ other statistical models. ANNs are the 
most used algorithms for energy consumption in buildings. In [10, it was used to predict energy consumption of a building simulation using EnergyPlus and presented a state-of-the-art result. They concluded that an ANN model with multiple outputs (larger number of neurons) has a better performance than an 30 ANN with single output. Also a study in [11] mentioned the use of Long Short Term Memory (LSTM) with Auto-Encoders (AE) in predicting solar energy consumption using weather data and achieved best results over several Deep Learning (DL) models. Furthermore in [12, different approaches were suggested based on the energy forecasting time range, where they suggested Neural Network based Genetic Algorithm (NNGA) for short and middle term prediction (for a day or a month) and Neural Network based Particle Swarm Optimization (NNPSO) for long term prediction (for a year). Both approaches were compared with the Conventional Neural Network (CNN) and performed better results.

Many studies have compared the uses of classification and regression algo40 rithms to Artificial Neural Network models in predicting energy consumption in buildings with varying results. In [13], ANN with two hidden layers and Back Propagation (BP) was compared to a decision tree algorithm (Random Forest $(\mathrm{RF}))$. ANN performed better results than RF. The data used was a combination of weather (dew point temperature, outdoor air temperature, wind 45 speed and humidity) and temporal information (month of the year, day of the week and hour of the day). In [14], ANN was tested along with SVM and times series algorithms. ANN and SVM showed adjacent results, but a better performance when combined together. While in [15], they reviewed different studies of hybrid approaches, where SVM, ANN and BPNN were combined with 50 Swarm Intelligence (SI) method. SVM showed better performance than ANN.

On the other hand, some studies showed that deep learning methods have outperform the traditional machine learning techniques. In [16], Multilayer 
Perceptron (MLP) was compared with Gaussian Processes, Support Vector Machines, Ensemble Boosting, Linear Regression and Regression Trees for predicting aggregated energy demand and showed a state of art results. While in [17, MLP showed almost the worst performance in comparing with 7 different algorithms (ARIMA, MLR, SVR, BT, RF, KNN and MARS) to predict next day energy consumption, where SVR and RF showed the best results. In [18] different deep learning methods (DBN, MLP, ANN-NAR and ANN-NARX) were compared against traditional statistical learning models (SVM, HMM and FHMM) for building energy prediction, where DBM showed a robust performance in different scenarios followed by MLP.

In this paper, we have used the same dataset of [3], which contains data for around 4.5 months. In their study [3] they compared four different models: ${ }_{65}$ Linear Regression (LR), decision tree models: Gradient Boosting Method (GBM), RF and SVM, where GBM presented the best results. Our objective was to create an efficient data model based on MLP and compare it with the results of the four classification models, to achieve better performance, and to reach the optimum configuration for our model.

The rest of this paper is organized as follow: Section 2 describes our developed system, how we configured it and its functionality, Section 3 presents the dataset and types of information used in our experiment, Section 4 discusses the experiment and results, and Section 5 concludes the paper.

\section{Dataset}

The used dataset contains 35 different variables of weather information (temperature, humidity, pressure, wind speed, visibility, dew point), appliances and light energy consumption, and temporal data (Table 1). It was collected from an indoor and outdoor sensors network of a two storey building and a 
nearby airport. The building contains 10 temperature sensors (9 indoor and 1 outdoor) and 8 humidity sensors ( 7 indoor and 1 outdoor) (Figures 1 and 2 ). The weather station provides: temperature, humidity, visibility, pressure, wind speed and dew point temperature. The data was recorded every 10 minutes for 137 days, including the light energy, everyday consumption and the number of seconds from midnight.

We implemented the same setup as in 3], the data was split using CARET algorithm, $75 \%$ for training and $25 \%$ for testing. CARET creates a balanced split using a maximum dissimilarity approach [19]. The features were scaled between 0 and 1 with min-max normalization $x^{\prime}=\frac{x-\min x}{\max x-\min x}$, to ease the network training. Also in [3] they used BORUTA package [20] to identify the most important features, which defined the Number of Seconds from Midnight (NSM) and light energy as the most important variables in predicting the consumption. The dataset was tested on several scenarios by using all the features or by omitting some of them.

Table 1: Dataset variables

$\begin{array}{ll}\text { T1 Kitchen Temperature } & \text { RH1 Kitchen Humidity } \\ \text { T2 Living room Temperature } & \text { RH2 Living room Humidity } \\ \text { T3 Laundry room Temperature } & \text { RH3 Laundry room Temperature } \\ \text { T4 Office room Temperature } & \text { RH4 Office room Humidity } \\ \text { T5 Bathroom Temperature } & \text { RH5 Bathroom Humidity } \\ \text { T6 Temperature Outside } & \text { RH6 Humidity Outside } \\ \text { T7 Ironing room Temperature } & \text { RH7 Ironing room Humidity } \\ \text { T8 Teenager room Temperature } & \text { RH8 Teenager room Humidity } \\ \text { T9 Parents room Temperature } & \text { RH9 Parents room Humidity } \\ \text { To Weather Station Temperature } & \text { Weather Station Pressure } \\ \text { RHo Weather Station Humidity } & \text { Weather Station Windspeed } \\ \text { Weather Station Visibility } & \text { Weather Station Tdewpoint } \\ \text { Week status Weekday } & \text { Week status Weekend } \\ \text { Day of week Monday } & \text { Day of week Tuesday } \\ \text { Day of week Wednesday } & \text { Day of week Thursday } \\ \text { Day of week Friday } & \text { Day of week Saturday } \\ \text { Day of week Sunday } & \text { light energy } \\ \text { NSM Number of seconds from midnight } & \end{array}$




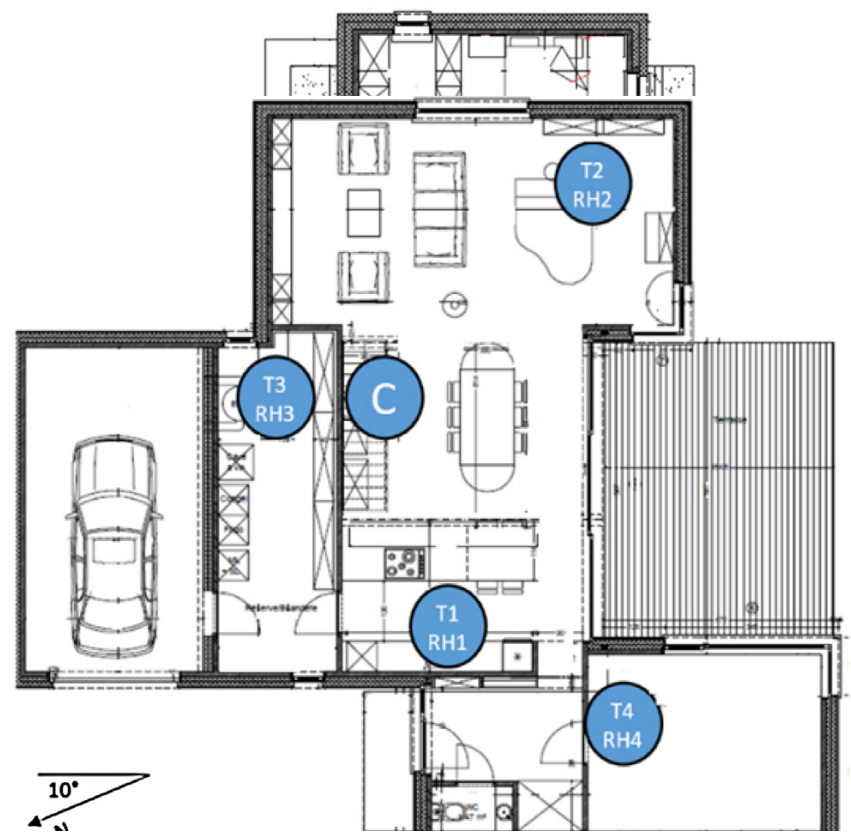

Figure 1: Building first floor 3

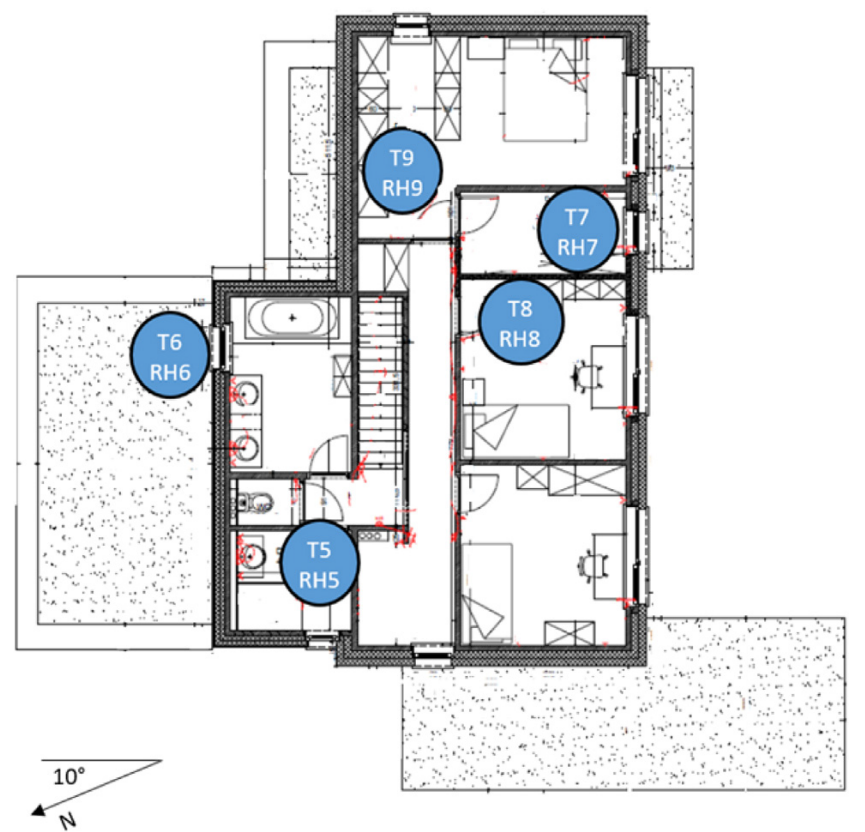

Figure 2: Building second floor 3] 


\section{System description} where the feature vector at the input layer (Table1) is mapped through a set of hidden layers to a single output representing the power consumption of the house (Figure 3). Each hidden layer in the network provides a specific representation of the input by building upon the output of the previous layer. With such level of abstraction, the network is able to build a robust representation of the input at high dimensions [21. The connection between two adjacent layers in the network can be defined as:

$$
\begin{gathered}
y_{j}^{n}=\sum_{i} h_{i}^{n-1} w_{i j}+b_{j}^{n} \\
h_{j}^{n}=f\left(y_{j}^{n}\right)
\end{gathered}
$$

105

where $y_{j}$ is the output of neuron $j$ at layer $n, h_{i}^{n-1}$ the activation of neuron $i$ at layer $n-1, w_{i j}$ and $b_{j}$ the weight and bias of the connection to neuron $j$ from the previous layer, and $f$ a nonlinear activation function.

\subsection{Non-Linear activation function}

We used the Rectified Linear Unit (ReLU) activation to achieve non-linearity; the function is used on the output of each neuron. It works by rectifying the input to 0 when $y<=0$ or by preserving the signal when it is positive.

$$
\begin{gathered}
h_{j}^{n}=\operatorname{ReLU}\left(y_{j}^{n}\right) \\
\operatorname{ReLU}=f(y)=\max (0, y)
\end{gathered}
$$

The ReLU activation function is simple in terms of computation. It solves the vanishing gradient problem, and works better than sigmoid and other activation functions. ReLU is considered the most recommended activation function for feed forward neural networks, as it helps to generalize a variety of non-linear data 22$]$. 


\subsection{Layer normalization}

The distribution of the output activation at each layer is subject to high change during training; which is known as the internal covariate shift. This instability in the input may allow the network to get stuck in a saturated mode, which would lead to slow convergence. Layer normalization is used to reduce the covariate shift at each layer in the network by fixing the mean and variance of the input. More specifically, the input is normalized across all features, regardless of the batch size. The mean $(\mu)$ and variance $(\sigma)$ across all hidden units at a given layer $l$ are computed as follow:

$$
\begin{gathered}
\mu^{l}=\frac{1}{H} \sum_{i=1}^{H} a_{i}^{l} \\
\sigma^{l}=\sqrt{\frac{1}{H} \sum_{i=1}^{H}\left(a_{i}^{l}-\mu^{l}\right)^{2}}
\end{gathered}
$$

where $H$ denotes the number of hidden units in a layer, and $a_{i}^{l}$ is the activation unit $i$ at a hidden layer $l$.

\subsection{Weight initialization}

The network weights allow for the preservation of a stable variance throughout the network layers. We initialized them as per [23] method, which improved the results and helped the network to converge faster. This method holds the signal from vanishing to zero or exploding to a high value. The weight matrices $W_{i j}$ were initialized with a uniform distribution given as $W_{i j} \sim U\left(-\frac{\sqrt{6}}{n}, \frac{\sqrt{6}}{n}\right)$, where $n$ is the total number of input and output neurons at the layer (assuming all layers are of the same size).

\subsection{Network training}

We train the network via gradient descent with mean square error (MSE) as loss function, which is the squared difference between the predicted power consumption and the expected output. Adam optimizer is used with initial 
learning rate of 0.005 and batch size of 500 . This algorithm is considered as an upgrade for RMSProp [24], which provides a bias correction and a momentum. Similar to Adadelta and RMSprop, it stores an exponentially decaying average of the past squared gradients and the past gradients (similar to momentum). It also offers a flexible learning rates for the stochastic gradient descent update, computed from the first and second moments of the gradients.

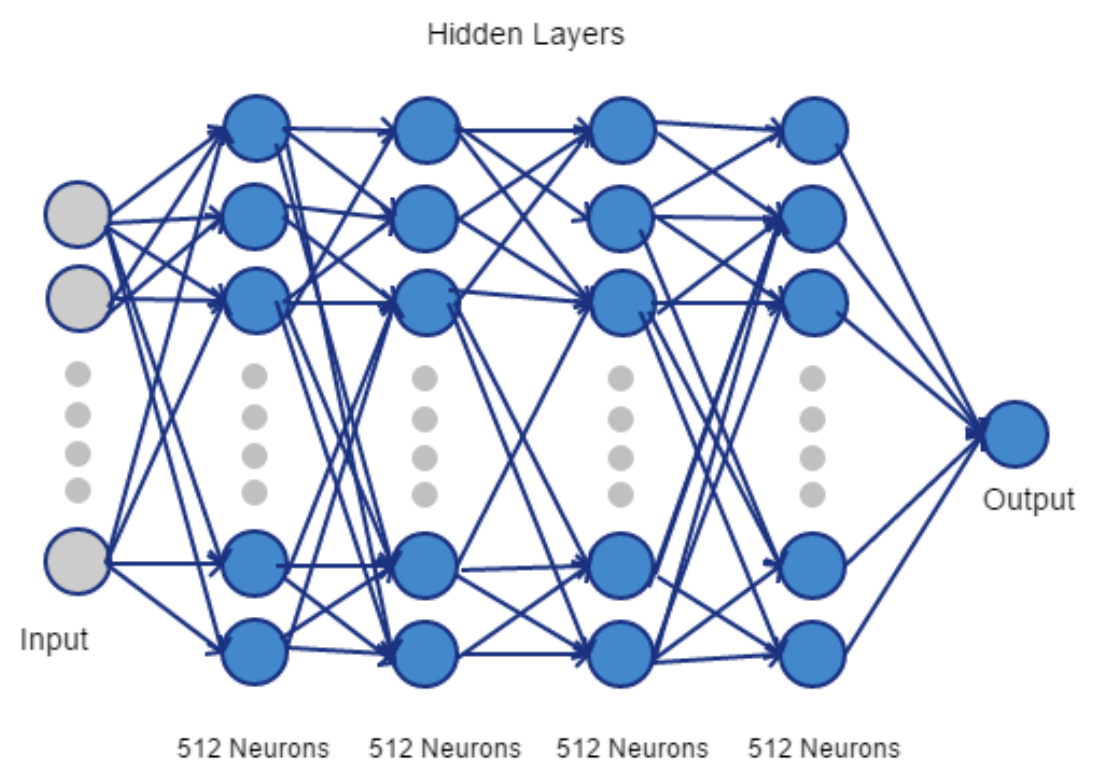

Figure 3: MLP with 4 hidden layers and back propagation

\section{Experiments and Results}

\subsection{Evaluation metrics}

We used four standard metrics to evaluate the performance of our system: Coefficient of determination $R^{2}$, Root Mean Square Error (RMSE), Mean Absolute Error (MAE) and Mean Absolute Percentage Error (MAPE). Their formulas are shown in Table 2 The $R^{2}$ is the proportion of variance between the predicted and testing variable, whereas RMSE is the percentage of difference between 
them. MAE is the percentage of difference between the predicted variables, while MAPE express the accuracy.

Table 2: Model evaluation criteria

\begin{tabular}{l|l}
\hline Coefficient of determination & $R^{2}=1-\frac{\sum_{t=1}^{n}\left(Y_{i}-Y_{i}^{\prime}\right)^{2}}{\sum_{t=1}^{n}\left(Y_{i}-Y_{i}^{\prime}\right)^{2}}$ \\
\hline Root mean squared error & RMSE $=\sqrt{\frac{1}{n} \sum_{t=1}^{n}\left(Y_{i}-Y_{i}^{\prime}\right)^{2}}$ \\
\hline Mean absolute error & MAE $=\frac{1}{n} \sum_{t=1}^{n}\left(Y_{i}-Y_{i}^{\prime}\right)$ \\
\hline Mean absolute percentage error & MAPE $=\frac{1}{n} \sum_{t=1}^{n}\left|\frac{\left(Y_{i}-Y_{i}^{\prime}\right)}{Y_{i}}\right|$ \\
\hline
\end{tabular}

\subsection{Hyperparameters optimization}

The selection of the number of layers and neurons in each layer can affect the model performance. To find the optimal parameters, we conducted a set of experiments with different configurations for the number of layers and neurons. In these experiments, all the features (weather, lights and temporal information) were used. To allow more generalization, we applied dropout [25] to the last hidden layer by randomly removing $50 \%$ of the neurons in that layer. However, we tested some models without dropout to access the improvements. Table 3 shows all the tested combinations, where $\mathrm{L}$ is the number of layers used in each model, $\mathrm{N}$ is the number of neurons per layer, DropOut shows the use of drop out at the last layer, Epochs is the number of complete passes through the data, and the four performance metrics represent the results. It is worth noting that layer normalization greatly improved network convergence. We noticed, for instance, a six fold increase in speed when training using layer normalization.

Our best system configuration is a MLP, with four hidden layers as illustrated in Figure 3. We used 512 neurons for each layer and we applied a dropout on the last layer. We also tried it without dropout and showed good results (a very slight difference), but we noticed a faster convergence when using dropout. The system needed a small number of Epochs to perform the best results, whereas, 
the second best configuration (3 hidden layers MLP without a dropout) used five times more epochs.

Table 3: Validating the best system configuration using all the data features

\begin{tabular}{llllllll}
\hline $\mathbf{L}$ & $\mathbf{N}$ & DropOut & Epochs & RMSE & R2 & MAE & MAPE \\
\hline 1 & 128 & Yes & 19149 & 77.234 & 0.408 & 43.354 & 48.365 \\
\hline 1 & 256 & Yes & 23425 & 74.285 & 0.450 & 41.870 & 46.829 \\
\hline 1 & 512 & Yes & 11178 & 73.549 & 0.462 & 39.793 & 43.019 \\
\hline 2 & 512 & Yes & 1940 & 69.415 & 0.526 & 34.570 & 35.714 \\
\hline 3 & 256 & Yes & 24563 & 67.298 & 0.541 & 31.892 & 31.640 \\
\hline 3 & 256 & No & 42610 & 68.889 & 0.534 & 32.293 & 31.999 \\
\hline 3 & 512 & Yes & 4778 & 67.182 & 0.550 & 31.281 & 30.860 \\
\hline 3 & 512 & No & 26748 & 66.245 & 0.567 & 30.599 & 29.160 \\
\hline $\mathbf{4}$ & $\mathbf{5 1 2}$ & Yes & $\mathbf{5 4 0 1}$ & $\mathbf{6 6 . 2 9 5}$ & $\mathbf{0 . 5 6 7}$ & $\mathbf{2 9 . 5 5 6}$ & $\mathbf{2 7 . 9 6 1}$ \\
\hline 4 & 512 & No & 14549 & 66.743 & 0.562 & 30.094 & 28.550 \\
\hline 5 & 512 & Yes & 3280 & 66.881 & 0.556 & 29.250 & 27.269 \\
\hline 5 & 512 & No & 12560 & 66.974 & 0.558 & 29.688 & 28.392 \\
\hline
\end{tabular}

\subsection{Comparative results}

After choosing the best configuration of layers and neurons, we tested our most performing MLP model with four different scenarios: 1) weather-only data which includes all the information from sensors inside the house and nearby airport station, 2) weather and lights information, 3) weather and temporal information (days of the week and Number of Seconds from Midnight (NSM)), and 4) all features data which includes weather, lights and temporal information. MLP yielded the best results with weather data and temporal information.

In [3], four algorithms were used to predict the power consumption: LR, RF, SVM, and GBM. The best model GBM was able to predict $57 \%$ of the variance 
$R^{2}$ with $66.65 \%$ RMSE, $35.22 \%$ MAE and $38.29 \%$ MAPE when using all the features. GBM performed its best with no lights and achieved slightly better results $58 \% R^{2}, 66.21 \%$ RMSE, $35.24 \%$ MAE and $38.65 \%$ MAPE, where the number of seconds till mid-night was considered the best feature to predict the consumption for all the algorithms.

In our experiments, MLP showed considerably better error rate $61.75 \%$ RMSE, an accuracy $R^{2} 61 \%, 28.52 \%$ MAE and $28.34 \%$ MAPE using only weather data. The importance of this result is that we omitted the most important feature (NSM) used by GBM in [3]. Our MLP achieved the best results at around $7 \mathrm{k}$ epochs. Table 4 shows the training (Trn) and Validation (Val) scores for the best scenario (without light energy variable). Figures 4, 5, 6 and 7 show the performance with respect to the four standard metrics.

We also tested the second best performing MLP (three hidden layers 512 neurons per layer without dropout) and showed high performance in the best scenario (using weather and temporal data): $63 \% R^{2}, 60.03 \%$ RMSE, $27.45 \%$ MAE and 26.85\% MAPE. Furthermore, we tried our best MLP configuration (four layers 512 neurons per layer) with a dropout on each layer to test its performance on same scenario (using weather and temporal data) and we got the following results: $57 \% R^{2}, 65.63 \%$ RMSE, $28.29 \%$ MAE and $25.51 \%$ MAPE.

Table 4: Training and validation scores for the best scenario

\begin{tabular}{llllll} 
& Epoch & RMSE & R2 & MAE & MAPE \\
\hline Trn & 7557 & 20.059 & 0.962 & 10.810 & 13.254 \\
\hline Val & 7557 & 59.840 & 0.643 & 27.283 & 27.096 \\
\hline
\end{tabular}




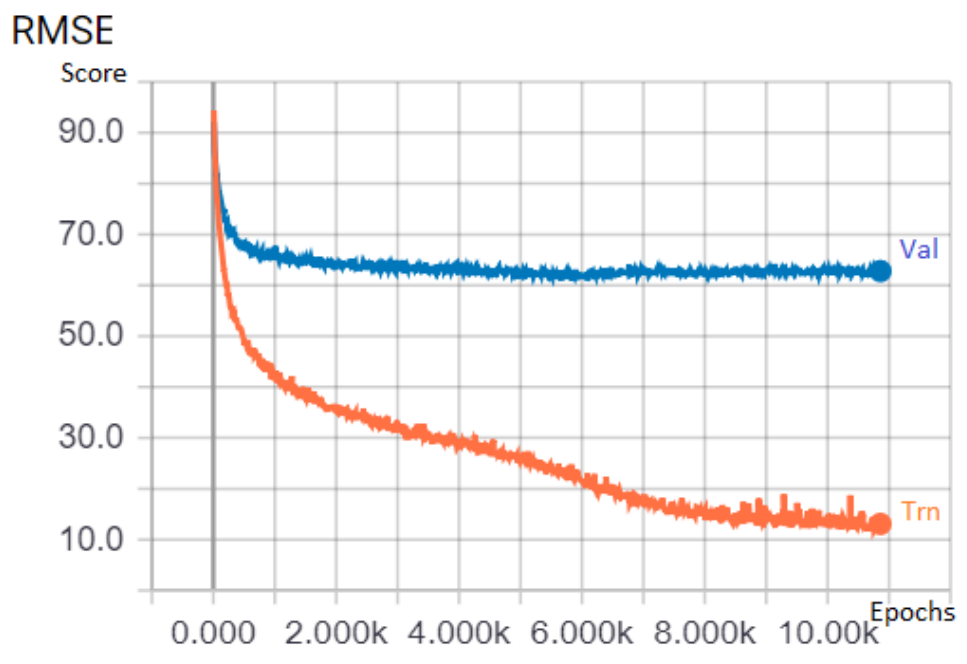

Figure 4: RMSE

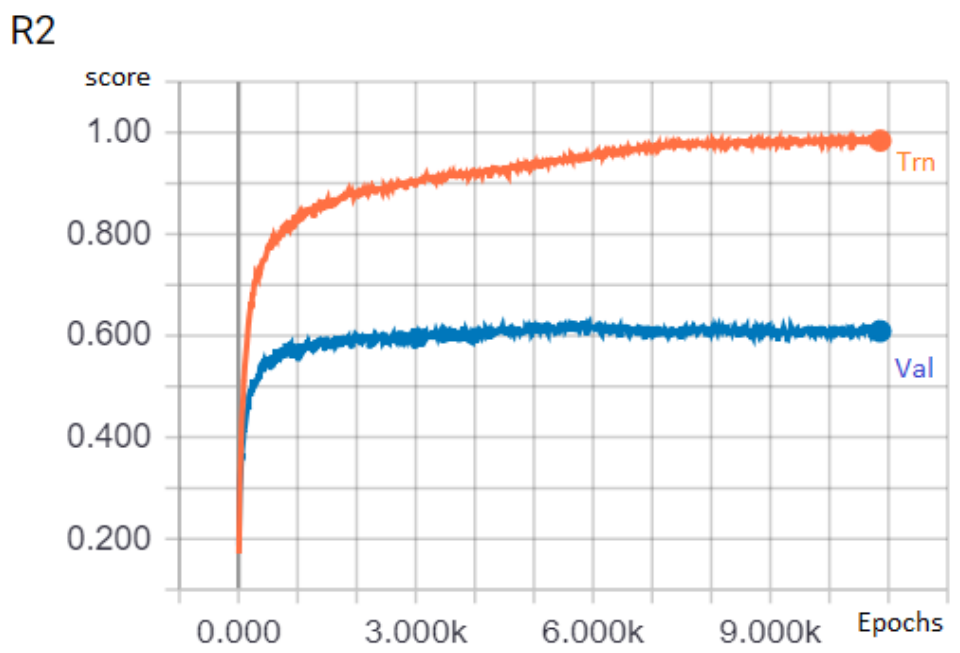

Figure 5: $R^{2}$ 


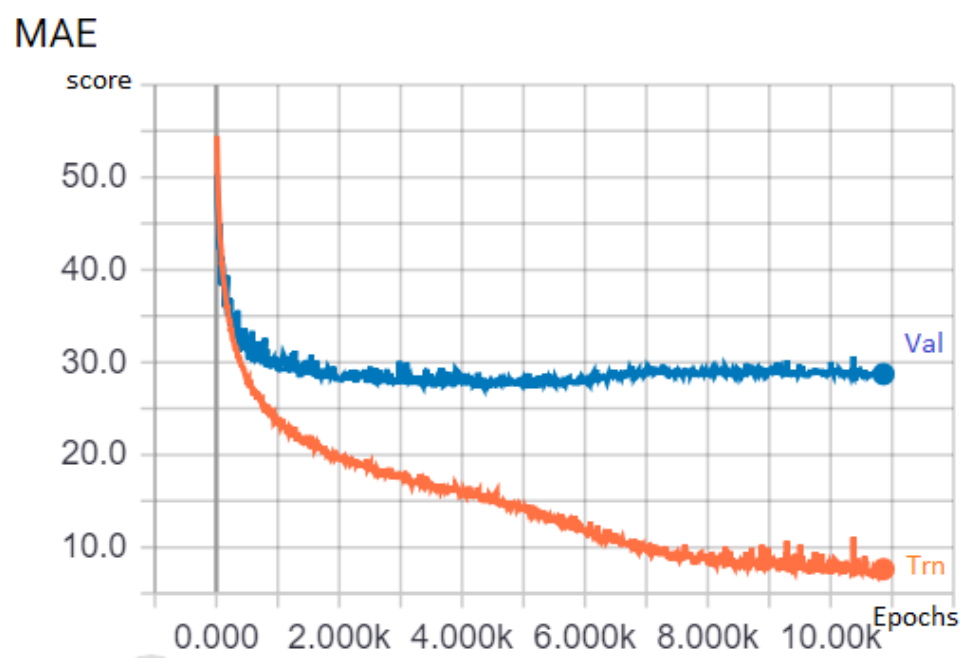

Figure 6: MAE

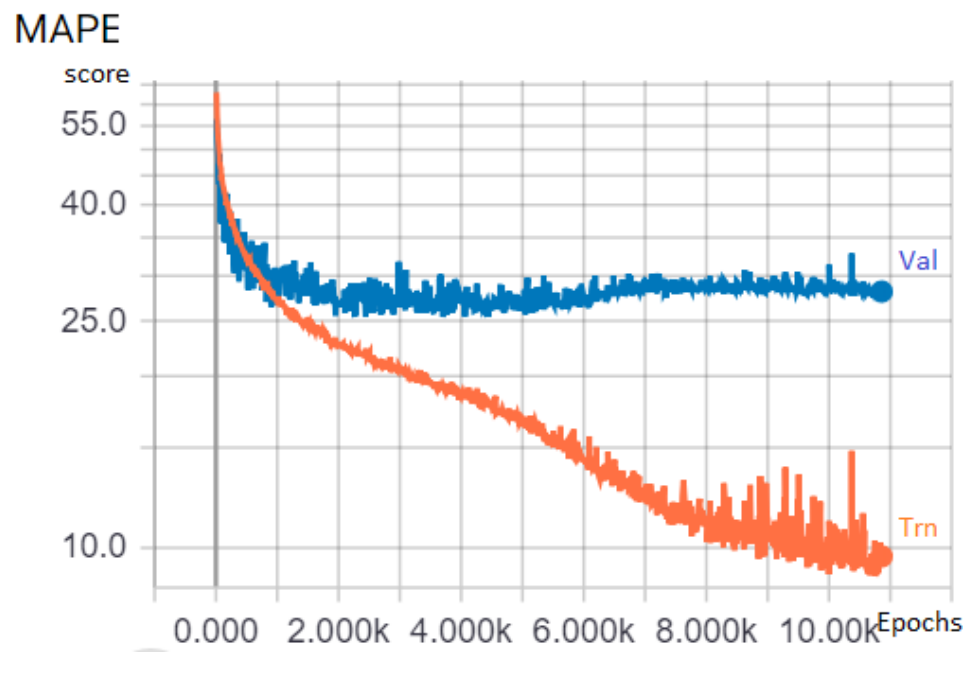

Figure 7: MAPE

The same experiment was repeated three times for MLP, first by adding the lights variable $\left(55 \% R^{2}, 67.34 \%\right.$ RMSE, $29.60 \%$ MAE and $28.03 \%$ MAPE), second by using all the features $\left(56 \% R^{2}, 66.29 \%\right.$ RMSE, $29.55 \%$ MAE and 215 $27.96 \% \mathrm{MAPE})$, and third by using weather and temporal information only, without lights ( $64 \% R^{2}, 59.84 \%$ RMSE, $27.28 \%$ MAE and $27.09 \%$ MAPE). The 
last experiment showed the best results with a slight improvement of around $2 \%$ and yielded the best performance. Table 5 shows the performance of MLP, in the four different scenarios, in comparison with other models on the same dataset. We notice that adding the temporal information has improved the results by $1 \%$ in all the experiments, whereas MLP looks more sensitive than GBM to lights feature which degraded the performance by $6 \%$.

Table 5: Models performance in the testing set

\begin{tabular}{ccccc}
\hline System & RMSE & $\boldsymbol{R}^{\mathbf{2}}$ & MAE & MAPE \% \\
\hline MLP weather only & 61.75 & 0.61 & 28.52 & 28.34 \\
MLP no date & 67.34 & 0.55 & 29.60 & 28.03 \\
MLP no lights & $\mathbf{5 9 . 8 4}$ & $\mathbf{0 . 6 4}$ & $\mathbf{2 7 . 2 8}$ & $\mathbf{2 7 . 0 9}$ \\
GBM no lights & 66.21 & 0.58 & 35.24 & 38.65 \\
MLP all features & 66.29 & 0.56 & 29.55 & 27.96 \\
GBM all features & 66.65 & 0.57 & 35.22 & 38.29 \\
RF all features & 68.48 & 0.54 & 31.85 & 31.39 \\
SVM all features & 70.74 & 0.52 & 31.36 & 29.76 \\
LR all features & 93.18 & 0.16 & 51.97 & 59.93 \\
\hline
\end{tabular}

\section{Conclusions}

One of the key factors for integrating systems and making homes and cities more intelligent is the energy savings that can be achieved. Therefore, energy prediction plays an important role in reducing power consumption. In this work, we propose to model a energy prediction system based on a Multilayer Perceptron neural network. Several data categories have been used, i.e.; light energy, temperature, humidity, day of the week, etc., collected from a Wireless Sensor Network installed in a two storey building. We compared the performance of our system against four classification algorithms, namely: Linear Regression, Support Vector Machine, Gradient Boosting Machine and Random Forest. We assessed the importance of temporal information and light energy as additional features for the prediction model. The temporal features slightly improved the 
performance for all systems, while the lights energy decreased the performance. For our method, we note a decrease by $10 \%$ in relative performance while the difference was negligible for the other classifiers (less than 1\% for Gradient Boosting Machine). Our system outperformed the other four classifiers in all scenarios. Based on the conducted experiments, we conclude that weather information would be enough to predict energy consumption. This enables lowcost solutions for energy predictions. Further experiments should be performed on different datasets to validate our results.

\section{References}

[1] P. V. Paul, R. Saraswathi, The internet of things - a comprehensive survey, in: Computation of Power, Energy Information and Commuincation (ICCPEIC), 2017 International Conference on, IEEE, 2017, pp. 421-426.

[2] J. Lee, J. Kim, D. Song, J. Kim, C. Jang, Impact of external insulation and internal thermal density upon energy consumption of buildings in a temperate climate with four distinct seasons, Renewable and Sustainable Energy Reviews 75 (2017) 1081-1088.

[3] L. M. Candanedo, V. Feldheim, D. Deramaix, Data driven prediction models of energy use of appliances in a low-energy house, Energy and buildings 140 (2017) 81-97.

[4] K. Amasyali, N. M. El-Gohary, A review of data-driven building energy consumption prediction studies, Renewable and Sustainable Energy Reviews 81 (2018) 1192-1205.

[5] H. Kaur, S. Ahuja, Time series analysis and prediction of electricity consumption of health care institution using arima model, in: Proceedings of 
Sixth International Conference on Soft Computing for Problem Solving,

260

[10] Q. Dong, K. Xing, H. Zhang, Artificial neural network for assessment of energy consumption and cost for cross laminated timber office building in severe cold regions, Sustainability 10 (1) (2017) 84.

[11] M. Mohammadi, A. Al-Fuqaha, S. Sorour, M. Guizani, Deep learning for iot

[12] K. Muralitharan, R. Sakthivel, R. Vishnuvarthan, Neural network based 
optimization approach for energy demand prediction in smart grid, Neurocomputing 273 (2018) $199-208$.

[19] P. Willett, Dissimilarity-based algorithms for selecting structurally diverse 
sets of compounds, Journal of Computational Biology 6 (3-4) (1999) 447457.

Michel Chammas received his M.S. degree in Computer Science - Networking and Communication from the University of Balamand, Lebanon, in 2009. Since 2010, he has been a Researcher at the Digital Humanities Center at the University 330 Internet of Things, Digital Humanities and CyberSecurity.

Abdallah Makhoul received the M.S. degree in computer science from INSA 
Lyon, France, in 2005, and the Ph.D. degree from the University of Franche335 Comté, Belfort, France, in 2008. Since 2009, he has been an Associate Professor with the University of Franche-Comté. His research interests include Internet of Things, structural health monitoring, and real-time issues in wireless sensor networks.

340 Jacques Demerjian received his PhD degree in Network and Computer Science from TELECOM ParisTech (Ecole Nationale Supérieure des Télécommunications - Paris) in 2004. Dr. Demerjian is a Professor at the Faculty of Sciences at the Lebanese University. His main research interests include Body Sensor Network, Mobile Cloud Computing and Recommender Systems. He is an IEEE Senior 345 Member. 